\title{
Evaluation of the COPDnet integrated care model in patients with COPD: the study protocol
}

This article was published in the following Dove Press journal:

International Journal of COPD

\author{
Eleonore H Koolen' \\ Philip J van der Wees ${ }^{2}$ \\ Gert PWestert ${ }^{2}$ \\ Richard Dekhuijzen' \\ Yvonne F Heijdra' \\ Alex J van 't Hul'
}

'Department of Pulmonary Diseases, Radboud University Medical

Center, Nijmegen, the Netherlands;

${ }^{2}$ Radboud Institute for Health

Sciences, IQ Healthcare, Radboud

University Medical Center, Nijmegen, the Netherlands
Correspondence: Eleonore H Koolen Department of Pulmonary Diseases, Radboud University Medical Center, PO Box 910I, 6500 HB Nijmegen, the Netherlands

Tel +3I $2436 \mid 0325$

$\mathrm{Fax}+3|2436| 0324$

Email noortje.koolen@radboudumc.nl
Background: Projections on the future suggest a further rise in the prevalence of patients with COPD, and in COPD related morbidity, mortality, and health care costs worldwide. Given the substantial impact on the individual and on society, it is important to establish a care process that maximizes outcomes in relation to the costs and efforts made. In an attempt to bridge this gap, we set out to develop an evidence-based model of integrated care for patients with COPD, named the COPDnet integrated care model.

Purpose: The current study protocol sets out to 1) evaluate the feasibility of employing the COPDnet model in present real-life care within the context of the Dutch health care system, 2) explore the potential health status benefits, and 3) analyze the costs of care of this model.

Patients and methods: In this prospective study, feasibility and health status changes will be evaluated with an experimental before and after study design. The costs of the diagnostic trajectory will be calculated according to a standard economic health care evaluation approach. Furthermore, the feasibility and cost of care studies will comprise both quantitative and qualitative data collection. For the studies on the feasibility and change in health status, all new patients qualifying for shared care by primary and secondary care professionals according to the Dutch Standard of Care for COPD, and patients referred by their general practitioners to one of the COPDnet hospitals will be included. To evaluate the feasibility and costs of care, semi-structured interviews will be held with patients, hospital personnel, health care professionals in the affiliated primary care region, and hospital and primary care group managers.

Conclusions: The COPDnet integrated care model for COPD patients has been designed according to the current insights regarding effective care for patients with a chronic condition in general, and for patients with COPD in particular. It will be evaluated for its feasibility, potential health status benefits, and the costs of care of the diagnostic trajectory in secondary care.

Keywords: COPD, COPD management, integrated care, chronic care model, health status

\section{Introduction}

COPD is defined as a common, preventable, and treatable disease that is characterized by persistent respiratory symptoms and airflow limitation. ${ }^{1}$ Moreover, COPD is a complex and heterogeneous disease with highly variable clinical manifestations. ${ }^{2,3}$ Even when COPD patients are in a relatively stable phase of their disease or only have mild-to-moderate airway obstruction, COPD often puts a significant burden of disease on those affected. ${ }^{2}$ The impact of COPD also places a high burden on health care resources, given the significant direct and indirect costs of care. ${ }^{4}$ Projections on the future suggest a further rise in the prevalence of patients with COPD, morbidity, mortality, and health care costs worldwide. ${ }^{5}$

Given the substantial impact on the individual and on society, it is important to establish a care process that maximizes outcomes in relation to the efforts and 
costs made. ${ }^{6}$ There is evidence to suggest that COPD disease management programs, providing integrated holistic care in an integrated care process, have the potential to better both health outcomes and costs compared with single-dimensional medical approaches. ${ }^{7}$ A recent review shows that better outcomes may be achieved with an integrated disease management program of at least 3 months duration with a follow-up ranging from 3 to 24 months on disease-specific quality of life and exercise capacity, and also, a reduction in respiratory-related hospital admissions and hospital days per person. ${ }^{8}$ The clinical impression is, however, that integrated care models are, as yet, only in limited use in our present care delivery pathways. This was confirmed in a recently performed survey in five European Union countries, including the Netherlands. In this paper, the authors concluded that COPD health care pathways are fragmented and care is not integrated properly. ${ }^{9}$

In an attempt to bridge this gap, we set out to develop an evidence-based model of integrated care for patients with COPD, named the COPDnet integrated care model. ${ }^{10}$ The COPDnet integrated care model has the ambition to innovate, simultaneously, both the content of care, ie applying a holistic approach, as well as the organization of care, ie, providing coherent, integrated care within the Dutch health care system. The use of such a model holds a promise to generate better outcomes of care for patients with COPD, as well as an overall reduction in the health care costs. ${ }^{11}$

As we acknowledge that it is a challenge to implement this highly complex COPDnet integrated care model, ${ }^{12}$ the current study protocol sets out 1) to evaluate the feasibility of employing the COPDnet model in present day real-life care within the context of the Dutch health care system, 2) to explore the potential health status benefits from the patients' perspective, and 3) to analyze the costs of care of this model.

\section{Objectives of the study protocol}

To realize the objectives of the current research project, a series of interrelated studies will be conducted with the following aims:

- To evaluate the feasibility of the implementation of the COPDnet integrated care model.

- To evaluate changes in health status over time in COPD patients who were offered care according to the COPDnet integrated care model.

- To evaluate the costs of care of the diagnostic trajectory in secondary care, as a component of the COPDnet integrated care model.
The objective of this article is to present the rationale and the different methods of this prospective study.

\section{Intervention}

The COPDnet integrated care model describes a transparent and highly standardized outpatient care process. Figure 1 represents a graphical overview of all elements of the COPDnet integrated care model, that is, a diagnostic trajectory carried out in secondary care, a non-medical intervention program provided in primary care, and a pulmonary rehabilitation service in tertiary care. The model also includes a quality management system based on patient-reported outcome measurements (PROMs), and regional appointments pertaining to exacerbation management and palliative care. Principles of the model include the following: 1) an individual care plan based on an assessment of the individual determinants of the burden of disease, 2) which is carried out closely to the natural habitat of the patient, and 3) looks for opportunities to initiate behavioral change, ie reinforcement of patients' self-management capabilities. Full details of the COPDnet integrated care model can be found in a recently published article by Koolen et al. ${ }^{10}$

\section{Methods and analyses Study setting and design}

The series of studies of this research project will be carried out in three hospitals and their affiliated primary care region, that is, the outpatient clinic of the Department of Pulmonary Diseases of Radboudumc (Nijmegen), Bernhoven Hospital (Uden), and Canisius Wilhelmina Hospital (Nijmegen).

In this prospective study, feasibility and health status changes will be evaluated with an experimental before and after study design. The costs of the diagnostic trajectory will be calculated according to a standard economic health care evaluation approach. Furthermore, the feasibility and cost of care studies will comprise both quantitative and qualitative data collection and analyses.

\section{Study population}

\section{Patients}

For the studies on the feasibility of the implementation of this COPDnet integrated care model and of the change in health status, patients will be included based on the following two criteria:

- New patients qualifying for shared care by primary and secondary care professionals according to the Dutch Standard of Care for COPD ${ }^{13}$

- Patients referred by their general practitioners (GPs) to the outpatient clinic of Radboudumc, Bernhoven Hospital, or Canisius Wilhelmina Hospital. 


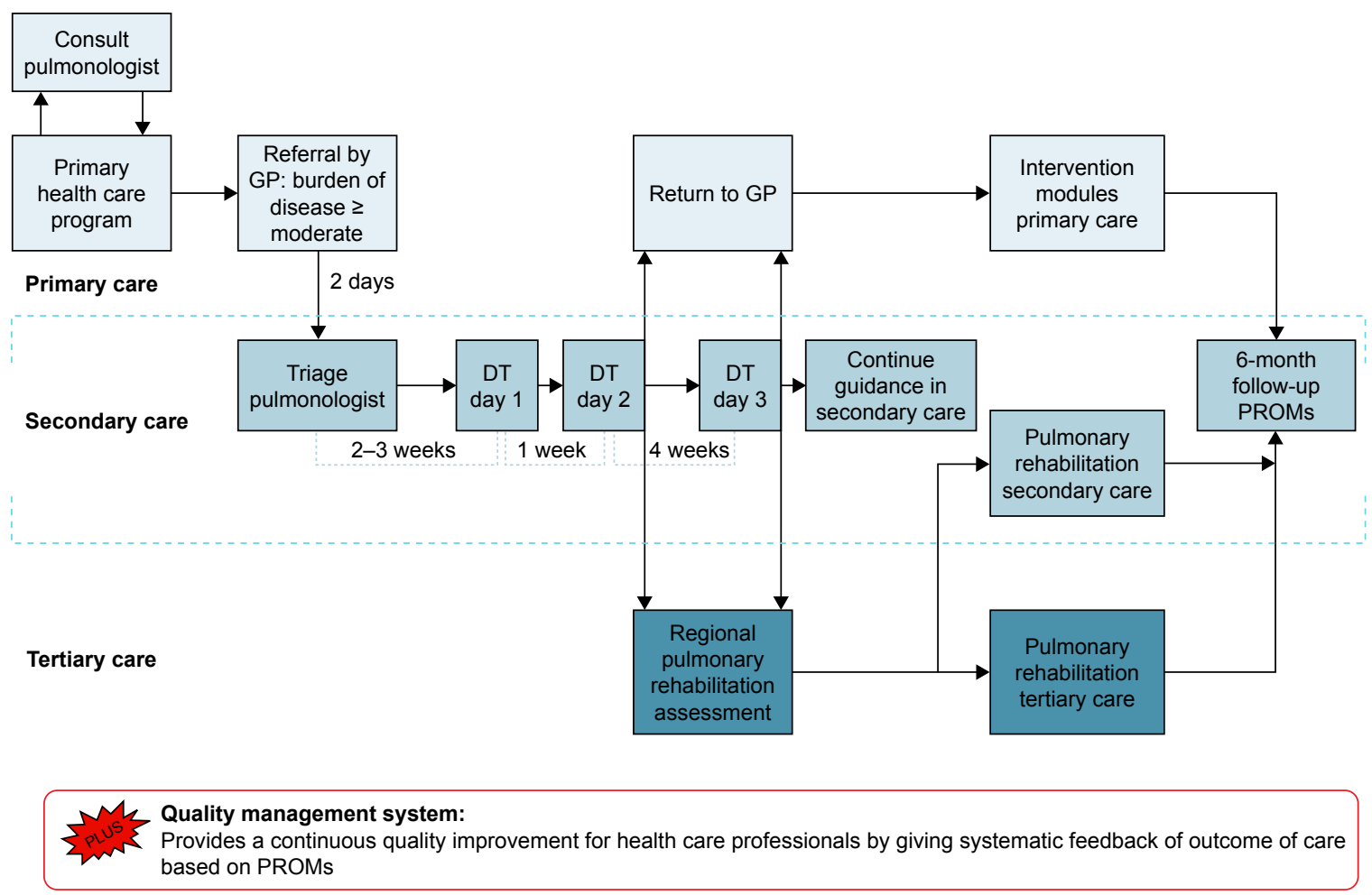

Figure I Graphical overview of the COPDnet integrated care model.

Abbreviations: DT, diagnostic trajectory; GP, general practitioner; PROMs, patient-reported outcome measurements.

\section{Patients will be excluded if}

- the patient is unable to complete questionnaires because of cognitive impairment and

- the patient is unable to speak or understand the Dutch language.

\section{Health care professionals}

To evaluate the feasibility and costs of care, the following health care professionals, providing care according to the COPDnet integrated care protocol, will be included in these studies:

- Primary care: GPs nurse practitioners, physiotherapists, dieticians, occupational therapists, and primary care group managers.

- Secondary care: pulmonary specialists, respiratory nurse specialists, pulmonary function technicians, medical secretaries, staff of the clinometric department, and hospital care group managers.

\section{Outcomes}

\section{Feasibility}

The feasibility of implementing the COPDnet integrated care model will be evaluated from three different perspectives, that is, patient perspective, health care perspective, and organizational perspective, applying both quantitative and qualitative methods.

\section{Quantitative evaluation}

The following questionnaires will be used for the evaluation of the patient outcomes: the Consumer Quality Index Asthma and $\mathrm{COPD}^{14}$ and the Patients Assessment Chronic Illness Care $^{15}$; to evaluate the health care professional perspective, the Assessment of Chronic Illness Care, ${ }^{16}$ the Clinician Support for Patient Activation Measure, ${ }^{17}$ and the Assessment of Primary Care Recourses and Supports for Chronic Disease Self-Management; ${ }^{18}$ and finally, for the evaluation of the organizational perspective, the Care Process Self Evaluation Tool will be applied. ${ }^{19}$

\section{Qualitative evaluation}

First of all, two authors (EHK and AJvH) will develop semi-structured interview guides, which should enhance the feasibility of using the COPDnet model in contemporary health care within the context of the Dutch health care system. The semi-structured interview guides will be tailored specifically to patients, hospital personnel, health care professionals in the affiliated primary care region, and hospital and primary care group managers. 
The patients will be selected based on the purposive sampling from the three COPDnet hospitals. We will seek a varied sample of patients based on age, sex, and burden of disease. The respiratory nurse specialists will invite these patients for participation in one of the interviews after they have completed the full trajectory of the COPDnet integrated care model. EHK will invite the professionals by telephone or e-mail. All participants will receive written information about the purpose and procedure of the interviews and they will be asked to confirm their participation by e-mail or telephone. Moreover, EHK will use the interview guides to conduct the semi-structured interviews, either by telephone or face-toface, after obtaining written informed consent.

Analysis of the patient perspective, health care professional perspective, and organizational perspective questionnaires, patient health records and the semi-structured interviews will be carried out between 12 and 36 months after the implementation of the COPDnet model in the three participating hospitals.

\section{Health status}

An analysis will be made of the potential improvements in health status through measuring changes in health status in patients enrolled in the COPDnet model, using widely accepted and validated PROMs. Primary outcome measure will be the Clinical COPD Questionnaire (CCQ). The results of the health status of enrolled patients at baseline, and at 6 months after the diagnostic trajectory in the COPDnet hospitals, will be compared. ${ }^{20}$ The CCQ is a self-administrated questionnaire and the psychometric properties are well documented.$^{20}$ In addition to the primary outcomes, the following secondary outcome measurements will be systematically registered in the COPDnet hospitals: the Patient Activation Measure, ${ }^{21,22}$ the Nijmegen Clinical Screening Instrument, ${ }^{23,24}$ and physical activity measured with the Marshall Questionnaire. ${ }^{25}$ All data will be collected at baseline during the first visit at the hospital, at discharge after completion of the diagnostic trajectory of the COPDnet model and, finally, after 6-month follow-up. Also, patient characteristics and the following health status characteristics will be registered at baseline: the medication prescribed in primary care, comorbidities, smoking behavior, pulmonary function, blood gas analysis, 6-minute walking distance, physical activity measured with an activity monitor, medical diagnosis, and the classification of the burden of disease.

\section{Costs of care}

Costs of care will be analyzed on the basis of completed and registered care activities performed during the diagnostic trajectory in the participating hospitals of the COPDnet model, complying with the principles of Time-Driven Activity-Based Costing (TDABC) in value-based health care. ${ }^{26} \mathrm{TDABC}$ requires the estimation of two parameters: the costs per time required to supply resources to the activities and the time required to perform activities in service deliveries. ${ }^{26}$ We will use the seven-step approach to the application of the TDABC in our health care setting. ${ }^{27}$ By using the TDABC model, we will be able to understand the cost of care delivery and control costs. ${ }^{28}$ We will develop TDABC models for all COPDnet hospitals, in which direct costs and indirect costs for delivering the diagnostic trajectory are taken into account.

Cost mapping will be performed on 1) calculation of the total (internal) costs of the COPDnet model on the basis of factual delivery of care activities and time spent to carry out these activities and 2) comparing the (internal) costs of the diagnostic trajectory with the reimbursement fees of the health insurance companies. Data source for cost mapping is the Hospital Information System of the participating hospitals.

TDABC models have been using different methods with respect to the time required to perform activities, which may result in different costs of patient care. ${ }^{28}$ Therefore, our TDABC models will be developed on the basis of three methods. First of all, we will evaluate the direct and indirect (non-) patient-related time through an objective measurement of time. Therefore, we will measure the time of each step in the diagnostic trajectory by using a stopwatch. Second, we will evaluate the direct and indirect (non-) patient-related time subjectively, by interviewing all the participating health care professionals. Third, we will interview the hospital care group managers, so that they may estimate the invested time of each step in the diagnostic trajectory in the secondary care setting. Differences and similarities between these three approaches will be evaluated. Finally, we will compare the TDABC models with the average costs of usual care, which was provided before the introduction of the COPDnet model, based on health care records.

\section{Qualitative evaluation}

EHK and AvtH will also develop the semi-structured interview guides for the participating health care professionals and hospital care group managers, in which the focus will be on an estimate of the time that the professionals have to invest to meet the requirements of the COPDnet model. All the participating health care professionals and hospital care group managers from the three COPDnet hospitals, who work according to the model, will be invited. EHK will invite the 
professionals by telephone or e-mail, and if they want to participate, they will receive written information about the purpose and procedure of the interview. EHK will use the interview guides to conduct the semi-structured interviews, either by telephone or face-to-face, after obtaining written informed consent.

\section{Sample size \\ Feasibility}

On each location, semi-structured interviews will be held with patients, clinicians, and managers. We anticipate on including five patients on each location - dependent on data saturation. Also, on each location, two pulmonary specialists, two respiratory nurses, and, of each discipline, two allied health care professionals will be invited to participate in the interviews. The to be interviewed pulmonary specialists and respiratory nurse specialists include all health care professionals, actually providing care following the COPDnet model in the three participating hospitals. Finally, a convenient sample of hospital and primary care group managers will be interviewed on what working with the COPDnet model means from an organizational perspective and how this affects feasibility.

\section{Health status}

The sample size for the secondary objective is estimated using G-power with an a priori paired $t$-test, based on an estimated difference between two dependent means. A total of 199 patients are required to detect a small effect size of 0.2 on the CCQ, with a significance of $5 \%$ and a power of $80 \%$. However, anticipating a dropout rate of $25 \%$, we will include 250 patients.

\section{Costs of care}

To evaluate the time invested, semi-structured interviews will be held with two pulmonary specialists, two respiratory nurses, one pulmonary function technician, one medical secretary, and one hospital care group manager, on each location.

\section{Recruitment}

All patients, qualifying for shared care in primary and secondary care settings (according to the Dutch Standard of Care for $\mathrm{COPD}^{13}$ ) and who are referred by their GP to secondary care will be automatically enrolled into the COPDnet model, in the outpatient clinic of the Radboudumc (Nijmegen), in the Bernhoven Hospital (Uden), or in the Canisius Wilhelmina Hospital (Nijmegen). Within these three hospitals, the COPDnet integrated care model has been accepted as the new standard care pathway for all new patients with COPD. All patients will be examined according to the new standard patient care pathway in one of the COPDnet hospitals mentioned above. Furthermore, patients will not be exposed to extra questionnaires, tests, or site visits. Only a minority of the purposively selected patients $(10-15 / 200=5 \%-8 \%)$, and all of the participating COPDnet health care professionals, will be asked to participate, voluntarily, in a semi-structured interview. Therefore, patients and health care professionals in this study will not be exposed to any additional health-related risk. Patients will be informed about the study by the way of a patient information form. All patients receiving usual care, according to the COPDnet model, will be included in these studies and will be excluded only when they say they are unwilling to participate.

\section{Data management}

All study data will be handled confidentially. The handling of the personal data will be fully compliant with the Dutch Personal Data Protection Act (de Wet Bescherming Persoonsgegevens). Study data will be kept for 15 years.

\section{Statistical analyses Quantitative analyses}

Descriptive statistics, including means, SD, frequencies, medians, and interquartile ranges will be used to summarize our collected data. Handling missing data will be carried out according to the guidelines of the different questionnaires.

To evaluate the feasibility of the COPDnet model, a two-tailed paired sample $t$-test or Wilcoxon signed rank test will be used to analyze the differences between the different measurement instruments at baseline and after implementation of the COPDnet model. To evaluate the potential health status benefits by testing the differences between baseline PROMS and follow-up PROMS, a two-tailed paired sample $t$-test or the Wilcoxon signed rank test will also be used. A priori, a two-sided level of significance will be set at $p \leq 0.05$. For data analysis, SPSS V.22.0 will be used.

The economic evaluation of the cost of care of the COPDnet model, based on the different TDABC models, will be analyzed with appropriate descriptive statistics and will be displayed graphically.

\section{Qualitative analyses}

To evaluate the feasibility and the costs of care, all semistructured interviews of the COPDnet model will be audio-taped after obtaining the respondent's permission. Subsequently, the audio tapes will be transcribed verbatim and entered into Atlas.ti qualitative software for analysis. 
We will use qualitative content analysis with a directed approach. ${ }^{29} \mathrm{We}$ will conduct a thematic analysis of the implementation of the COPDnet model. Results from the qualitative analyses will be triangulated with results from the quantitative analyses to identify concordant and disparate results across the data sources.

\section{Ethics and dissemination}

The Research Ethics Committee of the Radboud University Medical Center has passed a positive judgment on the study. The Committee considered that the study protocol does not fall within the remit of the Medical Research Involving Human Subjects Act (WMO) (ref: 2017/3597). Patients, health care professionals, and organizational managers will be asked to sign an Informed Consent form before participating in a semi-structured interview. This project will be conducted according to the Declaration of Helsinki and the Agreement on Medical Treatment Act (In Dutch: Wet op de Geneeskundige Behandelingsovereenkomst or WGBO).

\section{Discussion}

This study protocol focuses on the challenge to implement the COPDnet integrated care model into three hospitals and affiliated primary care regions. It will evaluate the feasibility of integrating the COPDnet model into actual care within the context of the Dutch health care system. It will evaluate the potential health status benefits from the patient's perspective, and it will evaluate the direct and indirect costs, on the basis of TDABC models, of the diagnostic trajectory as a component of the COPDnet model. This study protocol has strengths and limitations, which will be discussed below.

\section{Strengths}

A major strength of this study protocol is that we evaluate Dutch real-life care, derived from the care delivered by the implemented COPDnet integrated care models. Although the number of real-life studies on COPD is growing, ${ }^{30}$ little scientific data are available on the outcome of real-life COPD care outside the remit of treatment or exacerbations. ${ }^{31}$ The first publications on the outcome of this real-life COPD care suggest room for improvement on the organization of care, the content of care, and the cost-effectiveness of care. ${ }^{9,32-35}$

This study will generate knowledge about the COPDnet model, which is founded on the principles of the CCM. The aim of the CCM is to transform daily care for patients with chronic illnesses from acute and reactive, to proactive, planned, and population-based. ${ }^{36}$ The use of the principles of the CCM in the context of COPD holds the promise to generate better outcomes of care. ${ }^{11}$

Furthermore, this study protocol describes a multisite study. The COPDnet model will be implemented in three hospitals, each with a different character, and, subsequently, in different allied primary care regions. Although the COPDnet integrated care model was designed for the Dutch health care system, it is deemed applicable, mutatis mutandis, in every country that sets out to apply an integrated approach in an integrated health care chain. We based the development of the COPDnet model on evidence and frameworks available in the international literature. We think that our model is applicable in other health care systems, when tailored to national and local context.

Finally, this study protocol will provide a multiperspective evaluation of the COPDnet model. Our COPDnet model is an integrated care model, and its evaluation is an integral part of it. Our analysis will focus on patient perspective, health care professional perspective, and organizational perspective in primary and secondary care settings.

\section{Limitations}

This study protocol has two limitations. First, this study protocol lacks a controlled design. This means that we have to be careful with the conclusions we draw from our study and we can apply the recommendations only to the local geographic context of our three COPDnet hospitals and primary care regions. However, randomized controlled trials may have a high internal validity, whereas real-life studies have high generalizability. ${ }^{30}$ Nevertheless, we consider the current study as a proof of concept of the deployment of the COPDnet model, that is of the feasibility of working with the model, rather than a study on its effectiveness. Second, we have developed the current COPDnet model as it stands, but we are aware of its fragility. After the diagnostic trajectory in a secondary care setting, patients may be referred for further guidance and intervention to either a primary care setting or a form of pulmonary rehabilitation in secondary or tertiary care settings. Referrals are based on the assessment of the burden of disease and actual individual traits. For this purpose, guidelines on decision making were developed. Even so, uptake, completion, and quality of the delivered intervention(s) can only be partly controlled for. As a result, changes in health status over time may be difficult to interpret.

\section{Clinical consequences}

This study protocol will provide prime insights with respect to feasibility, health status benefits, and cost of care of the 
diagnostic trajectory of the COPDnet integrated care model in three hospitals and their affiliated primary care regions. It is anticipated that adjustments of the current model are to be made. Subsequently, a follow-up study may be performed with a controlled design to evaluate the clinical (cost)effectiveness of the model.

\section{Conclusion}

The COPDnet integrated care model for patients with COPD has been designed according to current insights regarding effective care for patients with a chronic condition in general and for patients with COPD in particular. It will be evaluated for its feasibility, potential health status benefits, and the costs of care of the diagnostic trajectory in secondary care.

\section{Acknowledgments}

Jeanine Antons, Heleen van der Niet, and Jan Vercoulen are thanked for their valuable contributions during the designing process of the COPDnet integrated care model. Renata Straver is thanked for her linguistic support. This study was funded by unrestricted grants from AstraZeneca, Glaxo Smith Klein, Novartis, Chiesi, Takeda, Mundipharma, Teva, and Boehringer-Ingelheim.

\section{Disclosure}

The authors report no conflicts of interest in this work.

\section{References}

1. The Global Strategy for the Diagnosis, Management and Prevention of COPD, Global Initiative for Chronic Obstructive Lung Disease (GOLD) 2017. Available from: http://goldcopd.org/gold-reports-2017/. Accessed March 29, 2018.

2. Agusti A, Calverley PM, Celli B, et al. Characterisation of COPD heterogeneity in the ECLIPSE cohort. Respir Res. 2010;11:122.

3. Vestbo J, Agusti A, Wouters EF, et al. Should we view chronic obstructive pulmonary disease differently after ECLIPSE? A clinical perspective from the study team. Am J Respir Crit Care Med. 2014;189(9): 1022-1030.

4. Wouters EF. Economic analysis of the Confronting COPD survey: an overview of results. Respir Med. 2003;97(Suppl C):S3-S14.

5. Mannino DM, Buist AS. Global burden of COPD: risk factors, prevalence, and future trends. Lancet. 2007;370(9589):765-773.

6. Porter ME. What is value in health care? N Engl J Med. 2010;363(26): 2477-2481.

7. Boland MR, Tsiachristas A, Kruis AL, Chavannes NH, Rutten-van Molken MP. The health economic impact of disease management programs for COPD: a systematic literature review and meta-analysis. BMC Pulm Med. 2013;13:40.

8. Kruis AL, Smidt N, Assendelft WJ, et al. Integrated disease management interventions for patients with chronic obstructive pulmonary disease. Cochrane Database Syst Rev. 2013;10:CD009437.

9. Kayyali R, Odeh B, Frerichs I, et al. COPD care delivery pathways in five European Union countries: mapping and health care professionals perceptions. Int J Chron Obstruct Pulm Dis. 2016;11:2831-2838.

10. Koolen EH, van der Wees, PJ, Westert GP, Dekhuijzen PNR, Heijdra YF, van 't Hul AJ. The COPDnet integrated care model. Int JChron Obstruct Pulmon Dis. 2018;13:2225-2235.
11. Adams SG, Smith PK, Allan PF, Anzueto A, Pugh JA, Cornell JE. Systematic review of the chronic care model in chronic obstructive pulmonary disease prevention and management. Arch Int Med. 2007; 167(6):551-561.

12. Grol R, Grimshaw J. From bestevidence to best practice: effective implementation of change in patients' care. Lancet. 2003;362(9391):1225-1230.

13. Long Alliantie Nederland LAN. Zorgstandaard COPD [Care Standard COPD]. 2016. Available from: http://www.longalliantie.nl/ zorgstandaard-copd. Accessed March 29, 2018. Dutch.

14. Doorontwikkeling CQ-index Astma en COPD: validatie van een verkorte vragenlijst voor het meten van de kwaliteit van astma/COPD-zorg vanuit het perspectief van patiënten [Further development CQ-index Asthma and COPD, Validation of a shortened questionnaire for measuring the quality of asthma / COPD care from the perspective of patients]. Available from: https://www.nivel.nl/sites/default/files/bestanden/CQindex\%20Astma\%20en\%20COPD\%202014.pdf. Accessed March 29, 2018. Dutch.

15. Wensing M, van Lieshout J, Jung HP, Hermsen J, Rosemann T. The Patients Assessment Chronic Illness Care (PACIC) questionnaire in The Netherlands: a validation study in rural general practice. BMC Health Serv Res. 2008;8:182.

16. Bonomi AE, Wagner EH, Glasgow RE, VonKorff M. Assessment of chronic illness care (ACIC): a practical tool to measure quality improvement. Health Serv Res. 2002;37(3):791-820.

17. Hibbard JH, Collins PA, Mahoney E, Baker LH. The development and testing of a measure assessing clinician beliefs about patient selfmanagement. Health Expect. 2010;13(1):65-72.

18. Brownson CA, Miller D, Crespo R, et al. A quality improvement tool to assess self-management support in primary care. Jt Comm J Qual Patient Saf. 2007;33(7):408-416.

19. Vanhaecht K, De Witte K, Depreitere R, et al. Development and validation of a care process self-evaluation tool. Health Serv Manag Res. 2007; 20(3):189-202.

20. van der Molen T, Willemse BW, Schokker S, ten Hacken NH, Postma DS, Juniper EF. Development, validity and responsiveness of the Clinical COPD Questionnaire. Health Qual Life Outcomes. 2003;1:13.

21. Hibbard JH, Mahoney ER, Stockard J, Tusler M. Development and testing of a short form of the patient activation measure. Health Serv Res. 2005;40(6 Pt 1):1918-1930.

22. Hibbard JH, Stockard J, Mahoney ER, Tusler M. Development of the Patient Activation Measure (PAM): conceptualizing and measuring activation in patients and consumers. Health Serv Res. 2004;39(4 Pt 1): $1005-1026$

23. Peters JB, Daudey L, Heijdra YF, Molema J, Dekhuijzen PN, Vercoulen JH. Development of a battery of instruments for detailed measurement of health status in patients with COPD in routine care: the Nijmegen Clinical Screening Instrument. Qual Life Res. 2009;18(7):901-912.

24. Vercoulen JH. A simple method to enable patient-tailored treatment and to motivate the patient to change behaviour. Chron Respir Dis. 2012;9(4):259-268.

25. Marshall AL, Smith BJ, Bauman AE, Kaur S. Reliability and validity of a brief physical activity assessment for use by family doctors. $\mathrm{Br} J$ Sports Med. 2005;39(5):294-297; discussion 294-297.

26. Kaplan RS, Anderson SR. Time-driven activity-based costing. Harv Bus Rev. 2004;82(11):131-138, 150.

27. Kaplan RS, Porter ME. How to solve the cost crisis in health care. Harv Bus Rev. 2011;89(9):46-52, 54, 56-61 passim.

28. Keel G, Savage C, Rafiq M, Mazzocato P. Time-driven activity-based costing in health care: a systematic review of the literature. Health Policy. 2017;121(7):755-763.

29. Hsieh HF, Shannon SE. Three approaches to qualitative content analysis. Qual Health Res. 2005;15(9):1277-1288.

30. Saturni S, Bellini F, Braido F, et al. Randomized Controlled Trials and real life studies. Approaches and methodologies: a clinical point of view. Pulm Pharmacol Ther. 2014;27(2):129-138.

31. Porter ME, Larsson S, Lee TH. Standardizing patient outcomes measurement. N Eng J Med. 2016;374(6):504-506. 
32. Worth H, Buhl R, Criee CP, Kardos P, Mailander C, Vogelmeier C. The 'real-life' COPD patient in Germany: the DACCORD study. Respir Med. 2016;111:64-71.

33. Buhl R, Criee CP, Kardos P, et al. A year in the life of German patients with COPD: the DACCORD observational study. Int J Chron Obstruct Pulmo Dis. 2016;11:1639-1646.

34. Haughney J, Gruffydd-Jones K, Roberts J, Lee AJ, Hardwell A, McGarvey L. The distribution of COPD in UK general practice using the new GOLD classification. Eur Respir J. 2014;43(4):993-1002.
35. Ke X, Marvel J, Yu TC, et al. Impact of lung function on exacerbations, health care utilization, and costs among patients with COPD. Int JChron Obstruct Pulm Dis. 2016;11:1689-1703.

36. Coleman K, Austin BT, Brach C, Wagner EH. Evidence on the Chronic Care Model in the new millennium. Health Affairs. 2009;28(1):75-85.

International Journal of COPD

\section{Publish your work in this journal}

The International Journal of COPD is an international, peer-reviewed journal of therapeutics and pharmacology focusing on concise rapid reporting of clinical studies and reviews in COPD. Special focus is given to the pathophysiological processes underlying the disease, intervention programs, patient focused education, and self management protocols.

This journal is indexed on PubMed Central, MedLine and CAS. The manuscript management system is completely online and includes a very quick and fair peer-review system, which is all easy to use. Visit $\mathrm{http}: / / \mathrm{www}$.dovepress.com/testimonials.php to read real quotes from published authors. 